\title{
Team-Tipps
}

\section{Zuhören erhöht den Praxisgewinn}

\author{
Viele Ärzte verschenken rund 30\% Praxisgewinn, weil sie nicht auf die \\ Vorschläge aus ihrem Team hören. Zu diesem Schluss kommt ein \\ Betriebswirtschaftler, der die Daten von 1.500 Praxen ausgewertet hat.
}

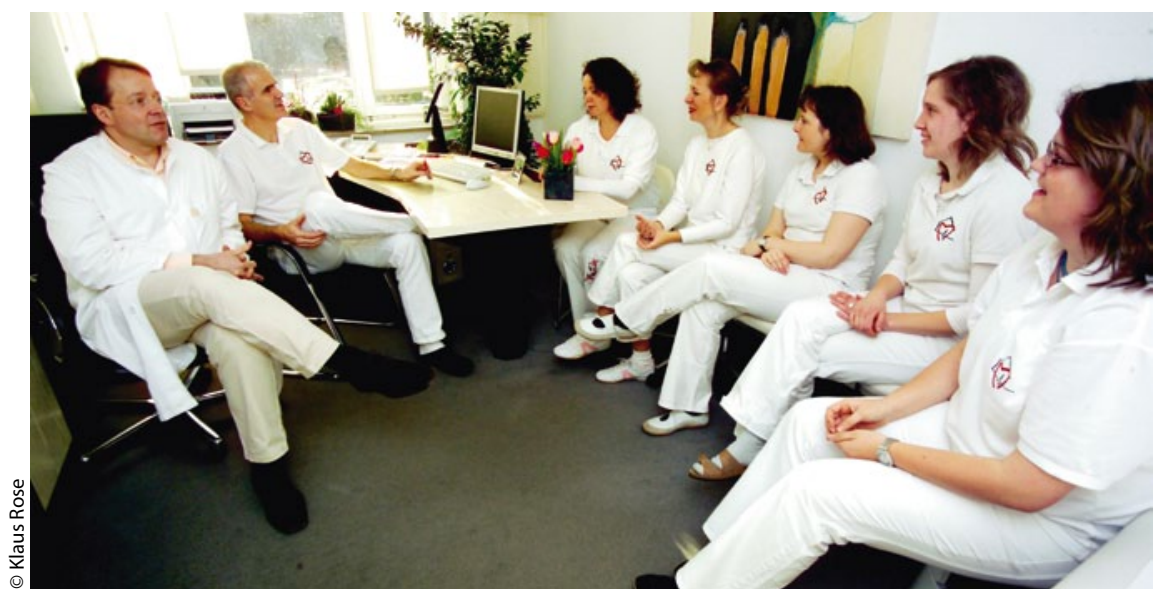

Regelmäßige Besprechungen mit den MFA gibt es nur in jeder dritten Praxis.

W enn niedergelassene Ärzte mehr auf ihre Patienten und ihre Mitarbeiterinnen hören würden, könnten sie sowohl ihre Arbeitsqualität als auch den Praxisgewinn erhöhen. Angesichts der Frustration über Faktoren wie das Vergütungssystem und die bürokratischen Belastungen vernachlässigen viele Praxisinhaber aber ihren individuellen Handlungsspielraum. „Sie kümmern sich zu wenig um das, was sie selbst tun können“, sagt Klaus-Dieter Thill, Leiter des Düsseldorfer Instituts für betriebswirtschaftliche Analysen, Beratung und Strategie-Entwicklung (IFABS). Thill hat 1.500 Praxisanalysen mit Blick auf die Praxisführung ausgewertet. Für vieles, was nicht optimal läuft, sind die Ärzte selbst verantwortlich, resümiert er. Der Grund: „Sie orientieren sich bei der Fehlersuche hauptsächlich an externen Einflüssen und kümmern sich kaum um die Möglichkeiten praxisinterner Veränderungen und Verbesserungen." Außerdem gingen sie zu häufig davon aus, dass in der Praxis schon alles gut laufe.

\section{Viele Praxen bestehen} Stresstest nicht hatten erst $12 \%$ der Inhaber die Praxisorganisation daraufhin überprüft, ob sie sich verbessern lässt. „Gleichzeitig gaben $68 \%$ der Mediziner an, dass die Abläufe bei größerer Belastung nicht mehr funktionsfähig seien“, so Thill. Ein wesentliches Manko sieht er darin, dass die Ärzte mit ihren Mitarbeiterinnen zu selten über praxisrelevante Dinge reden. So gibt es nur in $34 \%$ der Praxen regelmäßige Besprechungen.

Zwei Drittel der Medizinischen Fachangestellten (MFA) klagten über eine unzureichende interne Kommunikation und ihre negativen Folgen wie Doppelarbeiten oder Koordinationsprobleme, sagte der Praxisberater. „Viele Praxisinhaber schreiben ihren Mitarbeiterinnen in diesen Fragen zu wenig Kompetenz zu.“ Damit verschenkten sie viel Verbesserungspotenzial. Nach Angaben von Thill kannten die MFA bereits gut die Hälfte der Verbesserungsansätze, die das IFABS in den
Nach der Auswertung der Praxisanalysen
Praxen identifizierte. „Mit Verbesserungsvorschlag-Systemen könnte das Wissen nutzbar gemacht werden." Aber gerade einmal 5\% der Ärzte haben solche Systeme in ihren Praxen etabliert. Viele lassen noch eine wichtige Wissensquelle ungenutzt: die Patienten. In $48 \%$ der untersuchten Praxen gab es zwar Patientenzufriedenheits-Befragungen, aber nur in $16 \%$ wurden die Ergebnisse auch genutzt, sagt Thill.

\section{Geldwerte, aber ungenutzte Hinweise}

„Erhoben - ausgewertet - abgeheftet“, so kennzeichnet er den typischen Umgang mit den Befragungen. Das liege zum Teil daran, dass Ärzte die Patienten-Befragungen als eine Anforderung des Qualitätsmanagements sehen, die sie erfüllen müssen, mit der sie sich inhaltlich aber nicht auseinandersetzen. Dabei enthielten gerade die freien Äußerungen der Patienten großes Potenzial - vor allem dann, wenn sie in Beziehung zu den Einschätzungen der Praxismitarbeiterinnen gesetzt werden.

Thill empfiehlt niedergelassenen Ärzten, für die Praxis einen Veränderungs- und Optimierungsplan aufzustellen. Er sollte auf drei Elementen fußen: der Auflistung der Stärken und Schwächen der Praxen aus Sicht des Arztes, der Vorschläge der MFA und der Anregungen von Seiten der Patienten. Mit einem solchen Plan könnten sie die Basis schaffen für zufriedenere Mitarbeiterinnen und Patienten, eine höhere Produktivität der Praxis, eine geringere Arbeitsbelastung und ein insgesamt besseres Betriebsergebnis. Thill schätzt, dass durch die Verhaltensänderungen eine Gewinnsteigerung um bis zu $30 \%$ möglich ist. Das Ganze verlange nicht viel mehr von den Ärzten, als aktiv zu werden. Das Engagement lohnt sich nicht nur unter finanziellen Aspekten, betonte er. „Es geht auch um den Abbau von Stress und Arbeitsbelastung sowie eine höhere Arbeitszufriedenheit."

Ilse Schlingensiepen 